\title{
Fibroblast contributes for osteoblastic phenotype in a MAPK- ERK and sonic hedgehog signaling-independent manner
}

\author{
Celio J. da Costa Fernandes ${ }^{1}$ - Augusto Santana do Nascimento ${ }^{1} \cdot$ Rodrigo A. da Silva $^{1}$. \\ Willian F. Zambuzzi ${ }^{1}$ (D)
}

Received: 9 February 2017/ Accepted: 30 May 2017/Published online: 3 June 2017

(C) Springer Science+Business Media New York 2017

\begin{abstract}
We hypothesized that a crosstalk between osteoblast and fibroblast (FB) exists, which contributes to bone as a dynamic tissue. Cell-free supernatants were harvested from fibroblast cultures and later subject preosteoblasts to investigate there capacity to modulate cell viability and differentiation mechanisms, reporting the possible involvement of Shh signaling as a paracrine mechanism. By exploring immunoblotting technology, we have shown that FB-released factors interfere with osteoblast metabolism by up-regulating the phosphorylation of FAK and Rac-1 proteins at the early stage and later contribute to osteoblast differentiation by up-modulating alkaline phosphatase (ALP) and in vitro mineralization. We also found that Shh signaling was not required during osteoblastic differentiation promoted by the FB-released factors as well as MAPK-ERK phosphorylation, while preosteoblast cultures subjected to osteogenic medium (O.M.) require downstream transducers of Shh, such as Patched and Gli-1, and MAPK-ERK. Altogether, our results indicate for the first time a possible mechanism involved in the crosstalk between fibroblasts and osteoblasts, as it was possible to observe trophic factors released by fibroblasts interfering decisively in osteoblast metabolism in a Shhindependent manner. This study collaborates the body of work that indicates paracrine signaling molecules participate in the crosstalk among bone-resident cells and explains, at least partially, the biological mechanisms
\end{abstract}

Willian F. Zambuzzi

wzambuzzi@ibb.unesp.br

1 Bioassays and Cell Dynamics Lab, Department of Chemistry and Biochemistry, Bioscience Institute, UNESP, Botucatu, SP, Brazil responsible for bone tissue dynamism, opening new avenues to understand etiologies of bone diseases.

Keywords Bone $\cdot$ Fibroblast · Osteoblast $\cdot$ Crosstalk $\cdot$ Cell signaling

\section{Introduction}

The perfect crosstalk among cells has been proved to be extremely decisive during bone development $[1,2]$ and responsive to physiologic requirements [3, 4]. In bone tissue, the crosstalk between the two main bone cells (osteoblasts and osteoclasts) has been well characterized [5]. It is known that bone marrow presents a wide variety of host interacting with each other, but very little information has been reported about the crosstalk between fibroblast (FB) and osteoblast [6]. As observed elsewhere, osteoblasts are cells originated from mesenchymal tissue and are nearly indistinguishable from fibroblasts [7-9].

In addition, morphogenetic proteins play important roles during tissue development, remodeling, and repair processes. Among others, bone morphogenetic protein (BMP) and Hedgehog $(\mathrm{Hh})$ members are extremely decisive for bone homeostasis [10-13], because they modulate and compromise cell differentiation at a bone marrow niche. Indian Hedgehog (Ihh) was found to be an important factor during mineralized tissue development. It has been proposed, using chick and mouse models, to be largely expressed in pre-hypertrophic chondrocytes [14].

In this present study, we examined if fibroblast is able to modulate osteoblast metabolism. Our main hypothesis tested was that fibroblast-released molecules modulate osteoblasts performance by requiring Sonic Hedgehog (Shh) signaling transducers, because hedgehog members 
are physiological factors that support tissue development and integrity. In order to address this issue, fibroblast cultures were conducted, and the fibroblast-conditioned medium was used to subject pre-osteoblast. First, our results showed that fibroblast-released molecules interfere in early pre-osteoblast metabolism and viability by upregulating both FAK and Rac-1 phosphorylations (up to $24 \mathrm{~h}$ ) and later contribute to osteoblast differentiation by up-modulating alkaline phosphatase (ALP) and in vitro mineralization. In addition, all stages assayed in this work showed to have a Shh-independent manner. In addition, we believe these results support better understanding of the relevance of the paracrine signaling between bone-resident cells, which open new avenues to understand bone disease etiologies, such as osteoporosis.

\section{Materials and methods}

\section{Antibodies}

The following antibodies were purchased from Cell Signaling (Danvers, MA, USA): Shh Antibody (\#2287, 19, 42, 45 kd), GLI Antibody (\#2553, 160 kd), Ptch 1 (\#2468, 180-210 kd), Integrin $\beta 1$ (\#34971, 115-135 kd), Rac1/ cdc42 Antibody (\#4651, $21 \mathrm{kd})$, Phospho-Rac1/cdc42 (Ser71) Antibody (\#2461, $28 \mathrm{kd}$ ), and GAPDH Rabbit (\#5174, 37 kd). From Abcam (Cambridge, MA, USA): Anti-Fak antibody (ab61113), anti-Fak (phpspho Y576 + Y577) antibody [EP1832Y] (ab76244), antiCofilin antibody (ab42824), anti-Cofilin (phospho S3) antibody (ab12866), Anti-ERK1 + ERK2 Antibody [ERK7D8] (ab54230), and Anti-ERK1/2 (phospho-Thr202/ Tyr204) antibody (ab214362).

\section{Cell cultures}

We used NIH-3T3-E1 (FB, fibroblasts) and (MC3T3-E1, subclone 4) pre-osteoblasts. During all experiments, the cells were maintained and cultured in Alpha-MEM medium containing antibiotics (100 U/ml penicillin, $100 \mathrm{mg} / \mathrm{ml}$ streptomycin), Ribonucleosides, and Deoxyribonucleosides, supplemented with $10 \%$ Fetal Bovine Serum (Nutricell, Campinas, SP, Brazil). Cells were maintained at $37{ }^{\circ} \mathrm{C}$ and $95 \%$ humidity, in $5 \% \mathrm{CO}_{2}$.

\section{Cell viability}

For the cell viability assay, the medium was conditioned for 3 days by the fibroblast cells and then used to subject the pre-osteoblasts, which were previously plated (96-well plates) at the density of $5 \times 10^{4}$ cells $/ \mathrm{ml}$. After $24 \mathrm{~h}$ exposed to the FB-conditioned medium, cell viability was assessed by the MTT assay, when MTT solution $(1 \mathrm{mg} / \mathrm{ml})$ was added and maintained in an incubator for an additional $3 \mathrm{~h}$. The MTT solution was removed, and $100 \mu \mathrm{l}$ of DMSO was added for solubilizing the dye formed by viable cells. Finally, the absorbance was measured at $570 \mathrm{~nm}$ using a microplate reader (SYNERGY-HTX multi-mode reader, Biotek, USA).

\section{Immunoblot}

After 3, 6, and $24 \mathrm{~h}$ of seeding (for adhesion test) or 10 days (for differentiation test), the cells were lysed [50 mM Tris- $\mathrm{HCl}, \mathrm{pH} 7.4,1 \%$ Tween 20, 0.25\% Sodium deoxycholate, $150 \mathrm{mM} \mathrm{NaCl}, 1 \mathrm{mM}$ EGTA, $1 \mathrm{mM}$ O-Vanadate, $1 \mathrm{mM} \mathrm{NaF}$, and protease inhibitors $(1 \mu \mathrm{g} / \mathrm{ml}$ aprotinin, $10 \mu \mathrm{g} / \mathrm{ml}$ leupeptin, and $1 \mathrm{mM}$ aminoethyl fluorosilicon 4-fluoride hydrochloride)] and the samples sonicated (1 pulse $\mathrm{s}^{-1}$-SONICS Vibra-Cell). The protein extracts were pooled by centrifugation, and the protein concentration determined by the Lowry method. To the extracts was added sample buffer [1:1 ratio, sample buffer: $2 \mathrm{X}$ sodium dodecyl sulfate (SDS), $100 \mathrm{mM}$ Tris- $\mathrm{HCl}(\mathrm{pH}$ 6.8), $200 \mathrm{mM}$ dithiothreitol (DTT), 4\% SDS, $0.1 \%$ bromophenol blue, and $20 \%$ glycerol].

\section{Alizarin red and alkaline phosphatase staining}

Pre-osteoblasts were plated $\left(5 \times 10^{4}\right.$ cells $\left./ \mathrm{ml}\right)$ in $24-w e l l$ plates and were treated at semi-confluence with the FBconditioned medium up to 10 days. The medium was changed every 3 days. ALP staining was used exactly as recommend by the manufacturer (SIGMAFAST BCIP/ NBT tablet). Previously, cells were fixed with $10 \%$ formalin for $1 \mathrm{~min}$, and then washed with Wash Buffer $\left(0.05 \%\right.$ Tween in $20 \mathrm{ml}$ in PBS free of $\mathrm{Ca}^{2+}$ and $\left.\mathrm{Mg}^{2+}\right)$. Afterwards, they were kept for $10 \mathrm{~min}$ in the dark in ALP solution, and the cells were washed with PBS and photographed in an inverted microscope. For Alizarin red $\mathrm{S}$ staining, the cells were fixed with $10 \%$ formalin for $30 \mathrm{~min}$ at room temperature, then $2 \%$ Alizarin-Red $\mathrm{S}$ dye was added to the cultures, and the plate was maintained into a dark chamber for $45 \mathrm{~min}$. Finally, the wells were washed with PBS, and the plate was photographed using an inverted microscope (Zeiss, Germany).

\section{Statistical analysis}

Results were represented as mean \pm standard deviation (SD). They were verified using student's $t$ test (2-tailed) with $p<0.05$ considered statistically significant and $p<0.001$ considered highly significant. In an experiment in which there were $>2$ groups, we used one-way ANOVA (non-parametric) with post-test of Bonferroni, in order to 

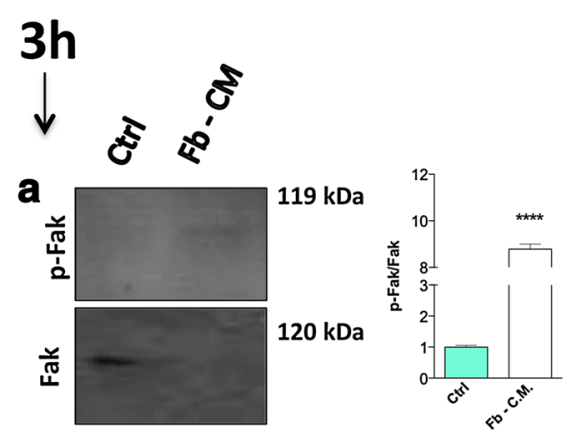

$6 \mathrm{~h}$

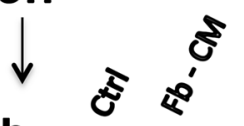

b

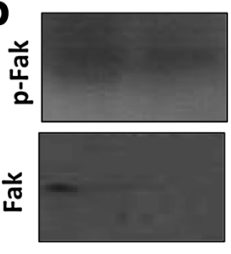

d

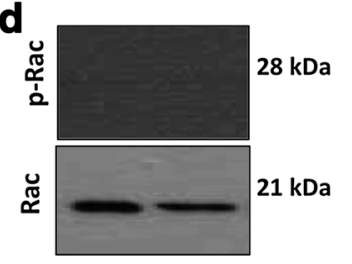

g

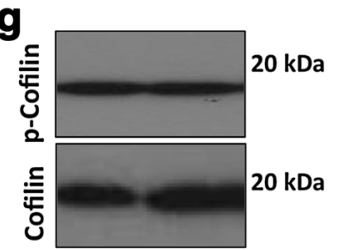

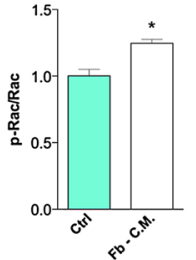

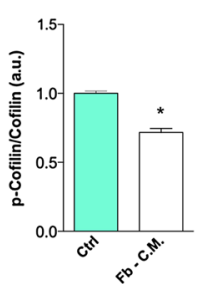

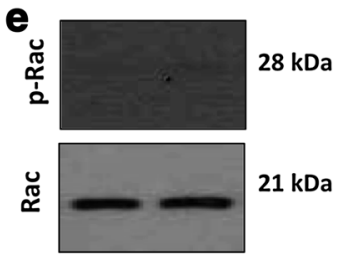

$119 \mathrm{kDa}$

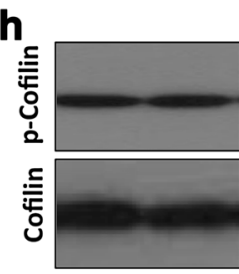

$120 \mathrm{kDa}$

$20 \mathrm{kDa}$
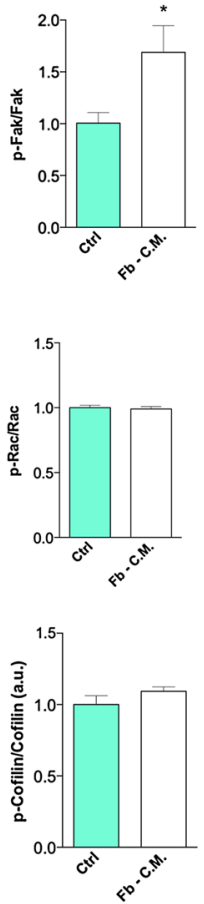

$24 \mathrm{~h}$
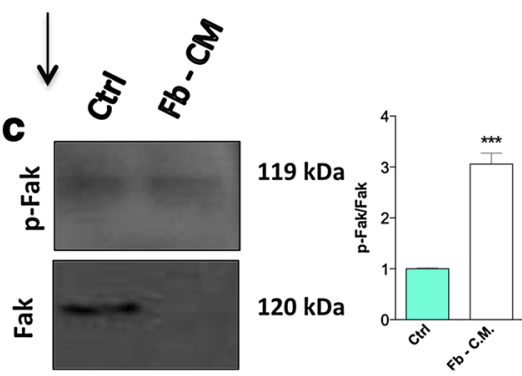

f
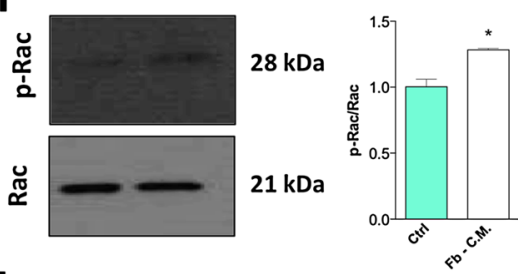

i

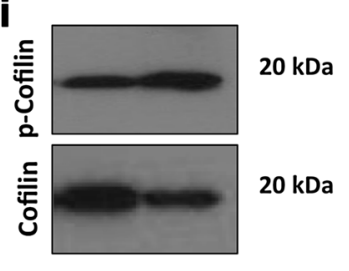

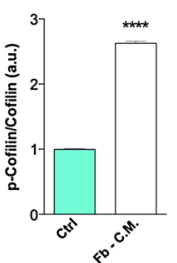

Fig. 1 Fibroblast interferes with osteoblast viability by modulating crucial signaling proteins. The cells were cultured under routine classic conditions. In the semi-confluence, the cells were treated with fibroblast-conditioned medium, and after $24 \mathrm{~h}$, the cells were trypsinized, counted, and re-seeded. After 3 (a, d, g), 6 (b, e, h), and $24 \mathrm{~h}(\mathbf{c}, \mathbf{f}, \mathbf{i})$ of seeding, the cells were lysed using standard lysing buffer (described in "Materials and methods"). The pooled protein was resolved on SDS-PAGE gel, and after PVDF membrane transfer.

compare all pairs of groups. In this case, the significance level was considered when alpha $=0.05$ (95\% confidence interval). The software used was GraphPad Prism 6.

\section{Results}

\section{Fibroblasts affect osteoblasts viability by modulating crucial signaling proteins}

As detailed in the "Materials and methods," fibroblasts were maintained up to $72 \mathrm{~h}$, and then the conditioned medium was collected to be used later. In the meantime, cultures of pre-osteoblasts (MC3T3-E1) were grown and, at the semi-confluent stage, were treated with the FBconditioned medium, as previously mentioned. After $24 \mathrm{~h}$, the pre-osteoblasts were trypsinized, counted, and re-plated. After 3, 6, and $24 \mathrm{~h}$ of seeding, the pre-
These were identified specifically by using specific primary antibody in Western blotting protocol. Representative blotting is shown, and the graphs represent arbitrary values obtained by densitometric analysis of bands normalized by the average values of the respective GADPH bands (housekeeping control). The differences were considered significant when $* p<0.01 ; \quad * * * p<0.0002 ; \quad$ and $* * * * p<0.0001$

osteoblasts were scraped in lysis buffer. These samples were resolved into SDS-PAGE approach for evaluating specific intracellular proteins using a specific antibody. We investigated crucial proteins involved in cell viability such as Rac-1, FAK, and Cofilin. Our results showed that FAK was progressively phosphorylated from 3 to $24 \mathrm{~h}$ (Fig. 1a-c), which suggests an effect of FB on preosteoblast adhesion mechanism and viability. In addition, Rac-1 phosphorylation was also reported, as presenting a significant profile at $24 \mathrm{~h}$ (Fig. 1d-f). Interesting, we also found that cofilin presented changeable phosphorylation profile: decreasing at $3 \mathrm{~h}$ (Fig. 1g) and significantly increasing at $24 \mathrm{~h}$ (Fig. 1i). Osteoblast metabolism was modified by FB-released factors requiring a transient cofilin phosphorylation at up to $24 \mathrm{~h}$ of treatment.

In addition, we observed few variations on the integrinB1 expression profile (Fig. 2), while the Patched-1 (Ptch) was significantly down-regulated in response to FB-released factors (Fig. 3). 


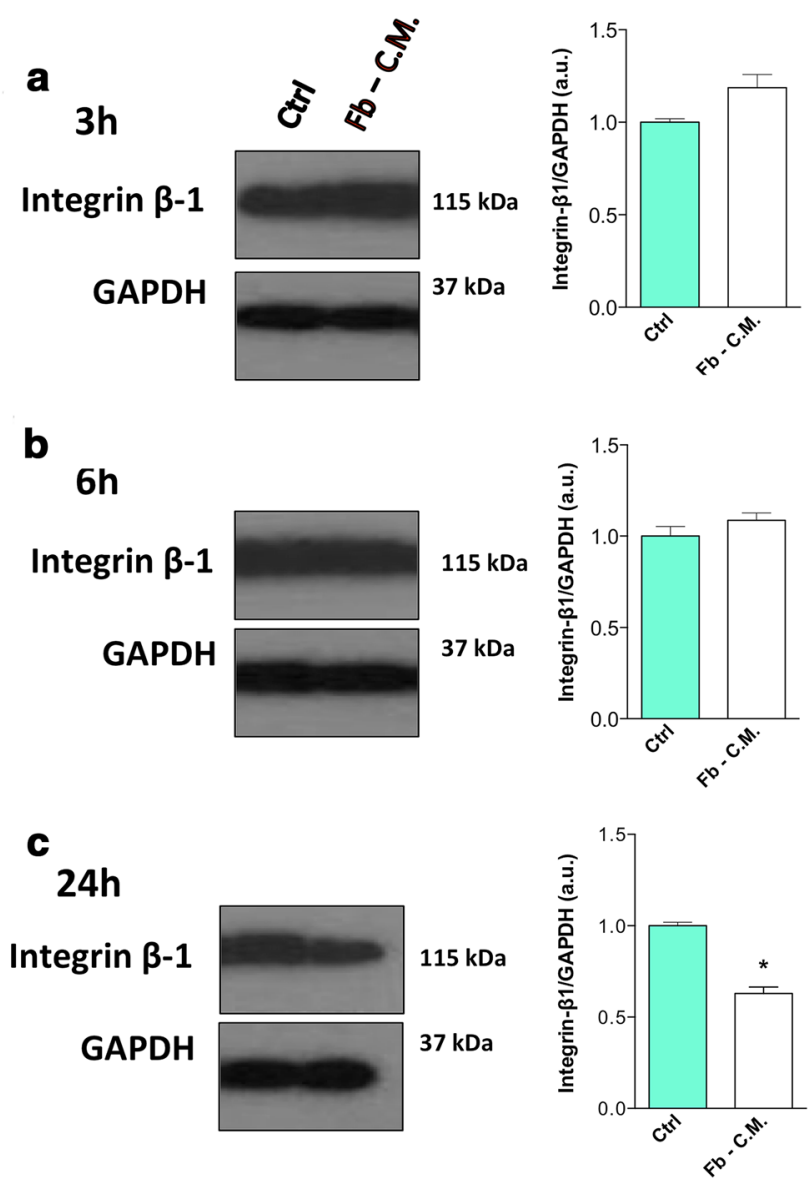

Fig. 2 Integrin- $\beta 1$ profile in response to fibroblast-conditioned medium. Briefly, the cells were cultured respecting the details listed previously. Representative blotting is shown and the graphs represent arbitrary values obtained by densitometric analysis of bands normalized by the average values of the respective GADPH bands (housekeeping control). The differences were considered significant when $* p<0.01$

\section{Fibroblasts contribute to osteogenic phenotype in a MAPK-ERK and Shh-independent manner}

Later, we evaluated the effect of the FB-conditioned medium on osteoblast differentiation up to 10 days by assaying 2 classical methodologies: Alkaline phosphatase (Fig. 4a-c); Alizarin red (Fig. 4d-f) staining. Our results showed that FB-conditioned medium was able to stimulate osteogenic differentiation because those two parameters were up-regulated (Fig. $4 \mathrm{~g}, \mathrm{~h}$ ) in response to FB-conditioned factors and when compared to 2 other control (Ctrl) groups. Two control groups were included in this experiment as follows: (1) Crtl: pre-osteoblasts were maintained under a classical condition; (2) Positive Ctrl: pre-osteoblast were subjected to osteogenic medium (O.M.), as described in "Materials and methods".

In addition, we investigated the involvement of sonic hedgehog signaling by evaluating Shh, Patched (Ptch), and
Gli-1 expressions by immunoblotting. Curiously, our results revealed that the O.M. promoted osteogenic phenotype acquisition by governing Shh signaling because Shh, Gli-1, and Ptch (Fig. 4i-n) were up-expressed. In contrast, FB-released factors also promoted osteogenic phenotype, but it was not dependent on Shh signaling, because Shh, Ptch, and Gli-1 were down-expressed (Fig. 4i-n). Moreover, MAPK-ERK involvement was also investigated, because MAPK-ERK has been reported to guide osteoblast differentiation. In this regard, our results showed that MAPK-ERK presented a different phosphorylation profile in response to O.M. and FB-released conditioned medium: while O.M. promoted an increase on MAPK-ERK phosphorylation, FB-released factors promoted its significant decrease (Fig. 4o, p).

\section{Discussion}

We are interested to understand how the trophic factors released from the host cells in the bone niche could be involved during osteoblastic phenotype. Here, we explored the possibility of fibroblast to modulate pre-osteoblast metabolism by investigating in vitro approaches. In order to explore this issue, first we collected conditioned medium from fibroblasts cultured up to 3 days and used to subject pre-osteoblast cells; thereafter, the subjected pre-osteoblasts were investigated regarding two important stages: adhesion (3, 6, and $24 \mathrm{~h}$ after seeding) and differentiation (up to 10 days in culture).

First, we found that FB modulated osteoblast viability by up-modulating crucial signaling proteins such as FAK and Rac-1 activations, during early stages of pre-osteoblast adhesion. These results are very interesting if we consider that both signaling proteins are involved with cytoskeleton rearrangement during pre-osteoblast adhesion $[15,16]$. The mechanism involving the synchrony of fibroblast/osteoblast crosstalk could enhance bone repair and might be involved during infectious-induced bone remodeling. In this regard, we reported elsewhere the attenuation of both FAK and Src phosphorylations in osteoblast during the response to TNF- $\alpha$ [17]. On the other hand, we can suppose that fibroblast-released factors could contribute to the osteoblast survival signaling, as it requires activation of FAK and Rac-1, while TNF- $\alpha$ has been suggested to attenuate both FAK and Src phosphorylations, culminating in impair of osteoblast performance. In addition, it is reasonable to suggest that the balance of the crosstalk between osteoblast and fibroblast, and considering the intensity of the TNF- $\alpha$ signaling, could guide the maintenance of the periodontal ligament healthiness and alveolar bone tissue repair, in bone commitment during periodontal diseases. FAK and Src activations are important parameters to 
Fig. 3 Patched is significantly down-regulated in crosstalking with fibroblasts. Briefly, the cells were cultured respecting the details listed previously. Representative blotting are shown, and the graphs represent arbitrary values obtained by densitometric analysis of bands normalized by the average values of the respective GADPH bands (housekeeping control). The differences were considered significant when $* * * p<0.0002$ and $* * p<0.0017$
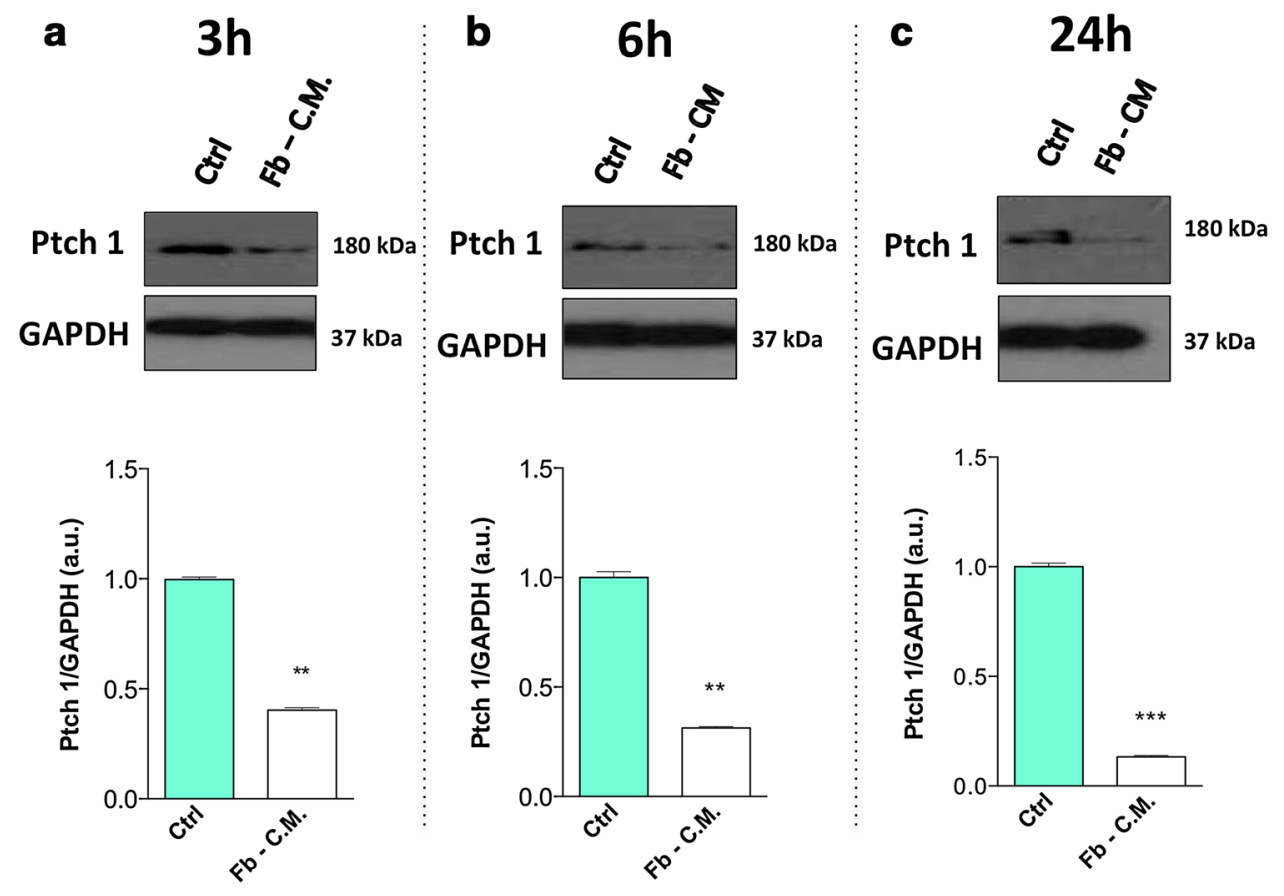

understand osteoblast phenotype in response to external stimuli [16, 18-20].

As reported, FAK and Src are very interesting biomarkers being considered in the paradoxical response of osteoblasts to TNF- $\alpha$ and fibroblast-released factors. As Protein Tyrosine Kinases, both FAK and Src are modulated by eventual Reactive Oxygen Species (ROS) because Protein Tyrosin Phosphatases are very sensitive to them. We have showed previously that Low Molecular WeightProtein Tyrosine Phosphatases (LMW-PTP) are able to modulate transient FAK and Src phosphorylations during pre-osteoblast adhesion [21]. In this regard, the modulation of upstream signaling transducers, such as FAK and Src, will certainly affect downstream molecules such as Rac-1, an important signaling transducer that directly guides cytoskeleton rearrangement-based pre-osteoblast adhesion by modulating cofilin phosphorylation.

Hence, we decided to also investigate the phosphorylation of cofilin (at serine 03), and thus, we found that during up to the first $24 \mathrm{~h}$ of seeding, cofilin phosphorylation was finely modulated, in agreement with the upstream signaling protein activation, such as Rac-1 and FAK. Thus, we believe FB starts modulating osteoblast phenotype by interfering with their viability and adhesion performance.

Thereafter, we investigated the influence of FB-released factors in contributing to osteoblastic phenotype. We showed that both alkaline phosphatase (ALP) and Alizarin red staining were up-modulated in response to FB-produced conditioned medium. In addition, we explored the dependency on canonical Sonic Hedgehog (Shh) signaling in this osteogenic phenotype acquisition by assessing Shh protein,
Gli-1, and Patched. Our results showed that FB-released factors promoted osteoblast differentiation in a Shh signaling-independent manner, as we found a decrease of Gli-1 and Patched in osteoblast metabolism in crosstalk with fibroblast. In contrast, osteogenic metabolism promoted by the classical osteogenic medium (used here as an internal control) required both Patch and Gli-1. Thus, we believe that several mechanisms are capable of promoting osteoblast differentiation and later mineralizing bone matrix.

Another protein evaluated in osteogenic phenotype acquisition was MAPK-ERK. Our results showed MAPKERK was up-activated in response to osteogenic medium, while differentiated osteoblast in response to FB-released factors did not present the same profile. MAPK-ERK is a signaling protein involved with a wide number of signaling pathways such as survival, proliferation, and differentiation [22, 23]. During the specific osteoblast differentiation, its involvement presents controversial results [24-29]. We consider these findings relevant because we suggest that the osteogenesis may come from a synergism of external trophic factors that require specific intracellular pathways.

Altogether, our results showed for the first time a possible mechanism involved in the crosstalk between fibroblasts and osteoblasts, resulting in the osteogenic phenotype at a Shh-independent manner. This collaborates to our understanding about paracrine signaling molecules that participate in the crosstalk among bone-resident cells, and thus explains, at least partially, biological mechanisms responsible for bone tissue dynamism, opening new avenues for comprehending bone diseases etiologies. 

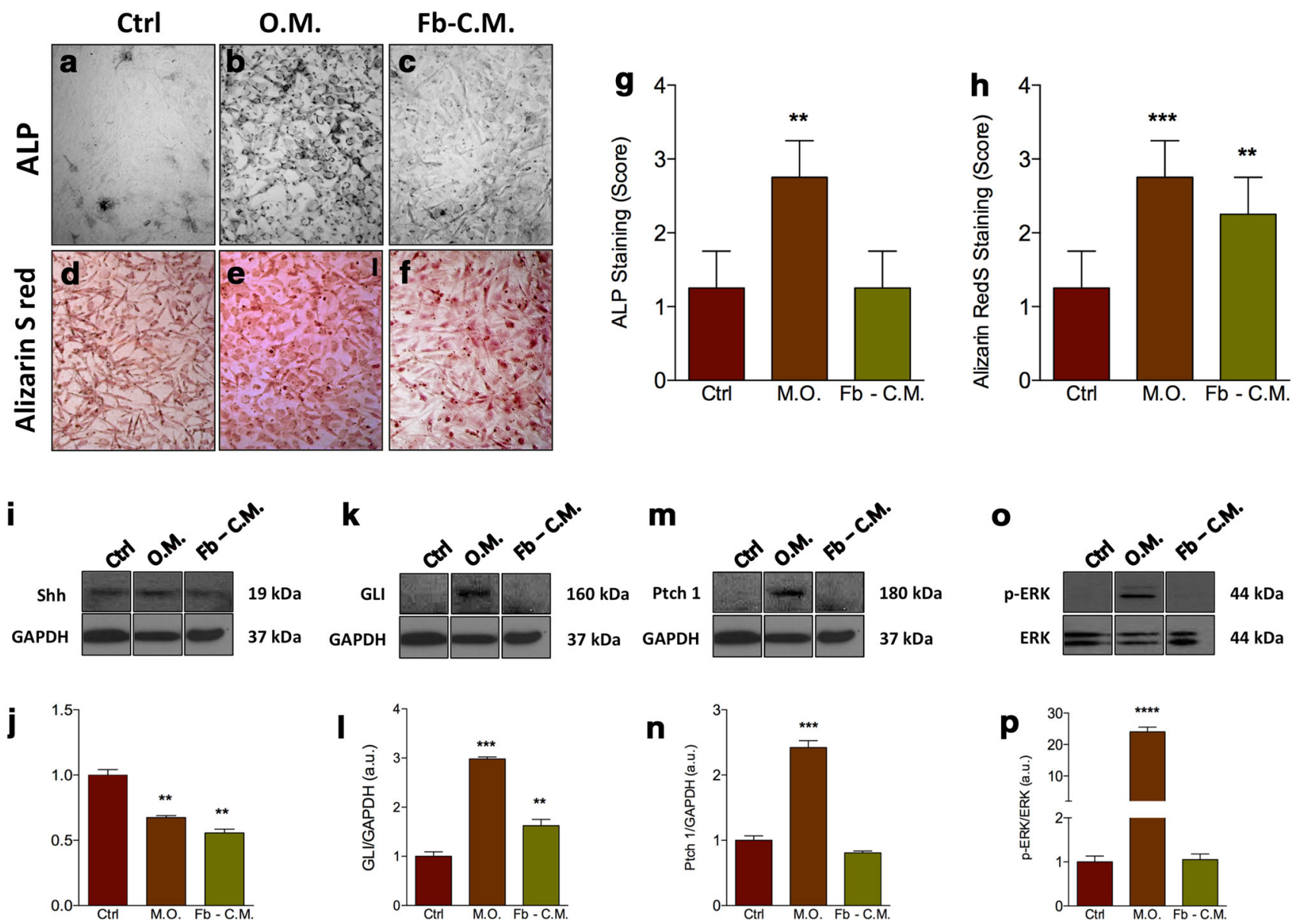

Fig. 4 Fibroblast paracrine effects promote osteoblast differentiation in a MAPK-ERK and Shh-independent manner. The pre-osteoblasts were cultured and in the semi-confluence were treated with conditioned medium from fibroblasts up to 10 days. Then the cells were subjected to osteoblast differentiation approaches: Alkaline phosphatase $(\mathbf{a}-\mathbf{c})$ and Alizarin red $(\mathbf{d}-\mathbf{f})$, which were scored, and the arbitrary values were attributed (g and $\mathbf{h}$, ALP and alizarin red,

Acknowledgements The authors are grateful to FAPESP (\#2015/ 00581-9; \#2014/22689-3) and CNPq (\#477452/2012-4) for the financial support. W.F.Z. is a PQ-2 fellow from CNPq (\#301966/2015-0).

Funding This study was funded by the Fundação de Amparo à Pesquisa do Estado de São Paulo - FAPESP (grants \#2015/00581-9; \#2014/22689-3) and the Conselho Nacional de Desenvolvimento Científico e Tecnológico - CNPq (grant \#477452/2012-4).

\section{Compliance with ethical standards}

Conflict of interest All the authors declare to have no conflict of interest with the materials used in the present study.

\section{References}

1. Ap Kusumbe, Sk Ramasamy, Adams Rh (2014) Coupling Of angiogenesis and osteogenesis by a specific vessel subtype in bone. Nature 507:323-328. doi:10.1038/Nature13145

respectively). For immunoblotting, the cells were cultured respecting the details listed previously. Representative blotting is shown $(\mathbf{i}, \mathbf{k}, \mathbf{m}$, $\mathbf{o})$, and the graphs $(\mathbf{j}, \mathbf{l}, \mathbf{n}, \mathbf{o})$ represent arbitrary values obtained by densitometric analysis of bands normalized by the average values of the respective GADPH bands (housekeeping control). The differences were considered significant when ${ }^{* * *} p<0.0002$ and $* * p<0.0017$

2. Sk Ramasamy, Ap Kusumbe, Adams Rh (2015) Regulation of tissue morphogenesis by endothelial cell-derived signals. Trends Cell Biol 25:148-157. doi:10.1016/J.Tcb.2014.11.007

3. Funck-Brentano T, Cohen-Solal M (2011) Crosstalk between cartilage and bone: when bone cytokines matter. Cytokine Growth Factor Rev 22:91-97. doi:10.1016/J.Cytogfr.2011.04. 003

4. Brotto M, Bonewald L (2015) Bone and muscle: interactions beyond mechanical. Bone 80:109-114. doi:10.1016/J.Bone.2015. 02.010

5. Gemini-Piperni S, Milani R, Bertazzo S, Al Et (2014) Kinome profiling of osteoblasts on hydroxyapatite opens new avenues on biomaterial cell signaling. Biotechnol Bioeng 111:1900-1905. doi:10.1002/Bit.25246

6. Ducy P, Schinke T, Karsenty G (2000) The osteoblast: a sophisticated fibroblast under central surveillance. Science 289:1501-1504

7. Lee B, Thirunavukkarasu K, Zhou L, Al Et (1997) Missense mutations abolishing dna binding of the osteoblast-specific transcription factor Osf2/Cbfa1 in cleidocranial dysplasia. Nat Genet 16:307-310. doi:10.1038/Ng0797-307 
8. Ducy P, Desbois C, Boyce B, Al Et (1996) Increased bone formation in osteocalcin-deficient mice. Nature 382:448-452. doi: $10.1038 / 382448 \mathrm{a} 0$

9. Ducy P, Zhang R, Geoffroy V, Al Et (1997) Osf2/Cbfa1: a transcriptional activator of osteoblast differentiation. Cell 89:747-754

10. Ac Carreira, Gg Alves, Wf Zambuzzi, Al Et (2014) Bone morphogenetic proteins: structure, biological function and therapeutic applications. Arch Biochem Biophys 561:64-73. doi:10.1016/J. Abb.2014.07.011

11. Gs Baht, Silkstone D, Nadesan P, Al Et (2014) Activation of Hedgehog signaling during fracture repair enhances osteoblasticdependent matrix formation. J Orthop Res 32:581-586. doi:10. 1002/Jor.22562

12. Krishnan L, Lb Priddy, Esancy C, Al Et (2017) Delivery vehicle effects on bone regeneration and heterotopic ossification induced by high dose Bmp-2. Acta Biomater 49:101-112. doi:10.1016/J. Actbio.2016.12.012

13. Lee J, Sk Madhurakkat Perikamana, Ahmad T, Al Et (2017) Controlled retention of Bmp-2-derived peptide on nanofibers based on mussel-inspired adhesion for bone formation. Tissue Eng Part A. doi:10.1089/Ten.Tea.2016.0363

14. Kazmers Nh, Ja Mckenzie, Ts Shen, Al Et (2015) Hedgehog signaling mediates woven bone formation and vascularization during stress fracture healing. Bone 81:524-532. doi:10.1016/J. Bone.2015.09.002

15. Wf Zambuzzi, Cl Yano, Adm Cavagis, Al Et (2009) Ascorbateinduced osteoblast differentiation recruits distinct Mmp-inhibitors: reck And Timp-2. Mol Cell Biochem 322:143-150. doi:10.1007/S11010-008-9951-X

16. Wf Zambuzzi, Bruni-Cardoso A, Jm Granjeiro, Al Et (2009) On the road to understanding of the osteoblast adhesion: cytoskeleton organization is rearranged by distinct signaling pathways. J Cell Biochem 108:134-144. doi:10.1002/Jcb.22236

17. Cavagis A, Takamori E, Granjeiro J, Al Et (2014) Tnfalpha contributes for attenuating both Y397fak And Y416src phosphorylations in osteoblasts. Oral Dis 20:780-786. doi:10.1111/ Odi. 12202

18. Milani R, Ferreira CV, Jm Granjeiro, Al Et (2010) Phosphoproteome reveals an atlas of protein signaling networks during osteoblast adhesion. J Cell Biochem 109:957-966. doi:10.1002/ Jcb. 22479

19. Wf Zambuzzi, Milani R, Teti A (2010) Expanding the role of Src and protein-tyrosine phosphatases balance in modulating osteoblast metabolism: lessons from mice. Biochimie 92:327-332. doi:10.1016/J.Biochi.2010.01.002

20. Wf Zambuzzi, Ferreira CV, Jm Granjeiro, Aoyama H (2011) Biological behavior of pre-osteoblasts on natural hydroxyapatite: a study of signaling molecules from attachment to differentiation. J Biomed Mater Res A 97:193-200. doi:10.1002/Jbm.A.32933

21. Gvo Fernandes, Adm Cavagis, Ferreira CV, Al Et (2014) Osteoblast adhesion dynamics: a possible role for Ros and LmwPtp. J Cell Biochem 115:1063-1069. doi:10.1002/Jcb.24691

22. Sun Z, Ss Guo, Fassler R (2016) Integrin-mediated mechanotransduction. J Cell Biol 215:445-456. doi:10.1083/Jcb. 201609037

23. Ohtsuka S, Ogawa S, Wakamatsu E, Abe R (2016) Cell cycle arrest caused by Mek/Erk signaling is a mechanism for suppressing growth of antigen-hyperstimulated effector $\mathrm{T}$ cells. Int Immunol 28:547-557. doi:10.1093/Intimm/Dxw037

24. Rutault K, Ca Hazzalin, Lc Mahadevan (2001) Combinations of Erk and P38 Mapk inhibitors ablate tumor necrosis factor-alpha (Tnf-Alpha) Mrna induction. Evidence for selective destabilization of Tnf-Alpha transcripts. J Biol Chem 276:6666-6674. doi:10.1074/Jbc.M005486200

25. Liu X, Zeng L, Zhao Z, Al Et (2017) Pbmc activation via the Erk and Stat signaling pathways enhances the anti-tumor activity of Staphylococcal enterotoxin A. Mol Cell Biochem. doi:10.1007/ S11010-017-3038-5

26. Guo J-P, Li X-G (2017) Galectin-7 promotes the invasiveness of human oral squamous cell carcinoma cells via activation of Erk and Jnk signaling. Oncol Lett 13:1919-1924. doi:10.3892/Ol. 2017.5649

27 Gao F, Li F, Miao Y, Al Et (2017) Involvement of the Mek-Erk/ $\mathrm{P} 38-\mathrm{Creb} / \mathrm{C}-\mathrm{Fos}$ signaling pathway in Kir channel inhibition-induced rat retinal muller cell gliosis. Sci Rep 7:1480. doi:10.1038/ S41598-017-01557-Y

28 Mc Moorer, Hebert C, Re Tomlinson, Al Et (2017) Defective signaling, osteoblastogenesis and bone remodeling in a mouse model of connexin 43 C-terminal truncation. J Cell Sci 130:531-540. doi:10.1242/Jcs. 197285

29 Wang M, Shu Z-J, Wang Y, Peng W (2017) Stachydrine hydrochloride inhibits proliferation and induces apoptosis of breast cancer cells via inhibition of Akt and Erk pathways. Am J Transl Res 9:1834-1844 